\title{
Global in Time Solutions to Kolmogorov-Feller Pseudodifferential Equations with Small Parameter*
}

\author{
S. Albeverio and V. G. Danilov
}

\begin{abstract}
The goal in this paper is to demonstrate a new method for constructing global-in-time approximate (asymptotic) solutions of (pseudodifferential) parabolic equations with a small parameter. We show that, in the leading term, such a solution can be constructed by using characteristics, more precisely, by using solutions of the corresponding Hamiltonian system and without using any integral representation. For completeness, we also briefly describe the well-known scheme developed by V. P. Maslov for constructing global-in-time solutions.
\end{abstract}

\section{Introduction}

The goal of the present paper is to present a new approach to the construction of singular (i.e., containing the Dirac $\delta$-function as a summand) solutions to the continuity equation and to show how these solutions can be used to construct the global in time solution of the Cauchy problem for Kolmogorov-Feller-type equations with diffusion, potential and jump terms. It is well known that the asymptotic solutions of the Cauchy problem for linear equations with a small parameter $\varepsilon>0$ can be constructed by the WKB method [27]. In the framework of this method, the initial partial differential equation is reduced to a system of equations consisting of the Hamilton-Jacobi equation and several transport equations. All these equations can be solved under the assumption that the Hamilton system has smooth solutions corresponding to the abovementioned Hamilton-Jacobi equation (the trajectories of the Hamilton system fiber the phase space). In general, this ensures only the existence of the classical solution in small with respect to time. In this case, if, for

\footnotetext{
${ }^{*}$ This work was supported by DFG project 436 RUS 113/895/0-1.
} 
example, the Hamilton function is time-independent, then, on the time intervals where the Hamilton-Jacobi equation has a smooth solution, the Cauchy problem for this equations (as well as for the corresponding transport equations) is invertible in time.

It is well known that the above system of equations (the HamiltonJacobi equation and several transport equations) arises in the construction of WKB solutions of the form $u_{\text {as }}=\exp \left\{\frac{i}{h} S(x, t)\right\} \varphi(x, t)$ for wave equations and Schrödinger-type equations [27] and in the construction of approximate solutions of the form $u_{\varepsilon}=\exp \left\{-\frac{1}{\varepsilon} S(x, t)\right\} \varphi(x, t)$ for parabolic equations [23].

We note that, in the first case (for stationary symbols), the property of being invertible in time is typical not only for solutions of this type but also for more general solutions because of the properties of the equation itself; but in the second case, the general solutions of the Cauchy problem do not have this property, and the fact that smooth "WKB-type" solutions $\exp \left\{-\frac{1}{\varepsilon} S(x, t)\right\} \varphi(x, t)$ are invertible in time distinguishes this class of solutions from the other solutions.

Now we explane the time-invertibility condition in more detail and describe (below in this paper) a method for constructing such solutions.

First, we note that the function $S(x, t)$ can be defined as the pointwise limit $S(x, t)=-\lim _{\varepsilon \rightarrow 0} \ln u_{\varepsilon}(x, t)$, where $u_{\varepsilon}$ is a solution of the equation of parabolic type with a small parameter [24].

If the function $S(x, t)$ thus defined exists and is smooth, then it is a solution (classical) of the Hamilton-Jacobi equation [24, 25]

$$
S_{t}+H\left(S_{x}, x\right)=0
$$

There is a well-known exact formula expressing the solutions of this equation in terms of the trajectories of the corresponding Hamilton system

$$
\dot{x}=H(p, x), \quad \dot{p}=-H_{x}(p, x) .
$$

The fact that the solution of Eq. (0.1) is smooth for $t=t_{0}$ means that the Lagrangian manifold $\Lambda_{t}^{n}$ obtained by a shift of the initial manifold $\Lambda_{0}^{n}=\left\{x,\left.\frac{\partial S}{\partial x}\right|_{t=0}\right\}$ along the trajectories of (0.2) can be uniquely projected on $R_{x}^{n}$ for all $t \in\left[0, t_{0}\right]$.

Since the shift along the trajectories of (0.2) is invertible in time, this implies that the resolving operator of Eq. (0.1) is also invertible in time. Under the same conditions of unique projection, the solution of the transport equation for the function $\varphi(x, t)$ (the amplitude) is given by the formula

$$
\varphi(x, t)=\frac{C}{\sqrt{J(x, t)}}
$$


where $C$ is a constant along the projection of the trajectories of $(0.2)$ on $R_{x}^{n}$ and $J(x, t)$ is the Jacobian of the mapping of shift along these projections (the uniqueness of the projection mapping implies the uniqueness and invertibility of the shift mapping).

Under the above conditions, formulas (0.3) are also invertible. Everything said above can be illustrated by the following simple example.

Let us consider the heat conduction equation

$$
\frac{\partial u}{\partial t}-\varepsilon \frac{\partial^{2} u}{\partial x^{2}}=0,\left.\quad u\right|_{t=0}=e^{-S_{0}(x) / \varepsilon} \varphi_{0},
$$

where the function $S_{0}(x) \geq 0$ is assumed to be smooth and bounded together with its derivatives and $\varphi \in C_{0}^{\infty}$, for example:

$$
S_{0}(x)=\int_{0}^{x}(1+\tanh z) d z=x+\ln \cosh x .
$$

Here the following addition condition is satisfied:

$$
\frac{d^{2} S_{0}}{d x^{2}} \geq 0
$$

The Hamilton function corresponding to $(0.3)$ is

$$
H(p, x)=p^{2}
$$

and, respectively, the trajectories of system (0.2) have the form

$$
x\left(x_{0}, t\right)=x_{0}+2 t p\left(x_{0}, t\right), \quad p\left(x_{0}, t\right) \equiv p\left(x_{0}, 0\right)=\frac{d S_{0}}{d x_{0}}=1+\tanh x_{0} .
$$

Obviously, condition (0.5) implies the unique globally-in- $t$ solvability of the equation

$$
x=x_{0}+2 t \frac{\partial S_{0}}{\partial x_{0}}
$$

in $\mathrm{i} x_{0}$ and hence the global solvability of the corresponding HamiltonJacobi equation and the transport equation in the class of smooth functions.

Further, for Eq. (0.3) it is possible to write the Green function, which is a solution of Eq. (0.4),

$$
G(x, \xi, t)=\frac{1}{\sqrt{2 \pi t \varepsilon}} e^{-(x-\xi)^{2} / 4 t \varepsilon},
$$

such that $G(x, \xi, 0)=\delta(x-\xi)$. It is well known that one can write

$$
u=\int G(x, \xi, t) u(\xi, 0) d \xi
$$


After the change $t \rightarrow-t$, we obtain the Green function for the inverse heat conduction equation, and it is easy to verify that

$$
\left.u\right|_{t=0}+O\left(\varepsilon^{N}\right)=\int G(x, \xi,-t) e^{-\hat{S}(\xi, t) / \varepsilon} \hat{\varphi}(\xi, t) d \xi
$$

where $\hat{S}(x, t)$ and $\hat{\varphi}(x, t)$ are solutions of the Hamilton-Jacobi equations and transport equation

$$
\frac{\partial \hat{\varphi}}{\partial t}+2 \frac{\partial S}{\partial x} \frac{\partial \hat{\varphi}}{\partial c}+\frac{\partial^{2} S}{\partial x^{2}} \hat{\varphi}=0
$$

at time $t$ satisfying the initial conditions

$$
\left.\hat{S}\right|_{t=0}=S_{0}(x),\left.\quad \hat{\varphi}\right|_{t=0}=\varphi_{0}(x),
$$

where $N>0$ is an arbitrary positive number and $O\left(\varepsilon^{N}\right)$ is such that

$$
e^{S_{0}(x) / \varepsilon} O\left(\varepsilon^{N}\right)=O\left(\varepsilon^{N}\right)
$$

Equality (0.7) can be verified using the Laplace method. The last equality means that the estimation $O\left(\varepsilon^{N}\right)$ is not quit suitable here.

There is an absolutely different situation if $(0.5)$ is replaced by the inequality

$$
\frac{d^{2} S_{0}}{d x^{2}}<0
$$

at least for a certain value of $x_{0}$, for example,

$$
S_{0}=\int_{0}^{x}(1-\tanh z) d z=x-\ln \cosh x .
$$

In this case, the jacobian of the mapping of shift along the trajectories $x=x_{0}+2 t \frac{d S_{0}}{d x_{0}}$ is zero at $t^{*}=\left(\min \left|2 \frac{d^{2} S_{0}}{d x_{0}^{2}}\right|\right)^{-1}$ (in the example, we have $\frac{d^{2} S_{0}}{d x_{0}^{2}}=-\cosh ^{-2}\left(x_{0}\right)$ and $\left.t^{*}=1 / 2\right)$, where the maximum is taken over all points at which the inequality $(0.8)$ is satisfied. In our example, this is $x_{0}=0$. For $t>t^{*}$, the Lagrangian manifold shifted along the trajectories (0.6),

$$
\Lambda_{t}^{1}=\left\{x=x_{0}-2 t\left(1-\tanh \left(x_{0}\right)\right), p=1-\tanh \left(x_{0}\right)\right\},
$$

forms an $S$-shaped curve, and there three points of this curve over a certain point $x$ in the plane $(x, p)$. These three points are associated with three (local) "WKB-type" solutions

$$
u_{j}=e^{-S_{j}(x, t) / \varepsilon} \varphi_{j}(x, t) .
$$


It is clear that their linear combination

$$
u=\sum_{j=1}^{3} c_{j} u_{j}
$$

is also a solution, i.e., satisfies the equation with the same accuracy as each of the functions $u_{j}, j=1,2,3$. But the functions themselves are not equivalent.

For example, it is clear that the inequality

$$
S_{1}(\bar{x}, t)>S_{2}(\bar{x}, t)
$$

holds at a certain point $\bar{x}$, then the "WKB" solutions $u_{1}$ and $u_{2}$ at the point $\bar{x}$ satisfy the relation

$$
\begin{aligned}
\left.u_{1}\right|_{x=\bar{x}} & =e^{-S_{1}(\bar{x}, t) / \varepsilon} \varphi_{1}(\bar{x}, t) \\
& =\left.e^{-S_{1}(\bar{x}, t) / \varepsilon} \varphi_{2}(\bar{x}, t)\left(e^{-\left(S_{1}-S_{2}\right) / \varepsilon} \varphi_{1} / \varphi_{2}\right)\right|_{x=\bar{x}} \\
& =u_{2} O\left(\varepsilon^{N}\right),
\end{aligned}
$$

where $N>0$ is an arbitrary number. This follows from the fact that the difference $\left.\left(S_{1}-S_{2}\right)\right|_{\bar{x}}$ in parentheses in the exponent is positive.

Thus, at each point in formula (0.9), it is necessary to choose the term where the function $S_{j}$ is minimal. Such a choice leads to an expression of the form

$$
u=e^{-\phi(x, t) / \varepsilon} \varphi(x, t),
$$

where $\phi=\phi(x, t)=\min _{x}\left\{S_{j}(x, t)\right\}$. It is clear that expression (0.11) is the leading term of the approximate solution. But its substitution into the equation in order to verify that it is a solution in a certain sense is not a trivial problem, because the function $\phi(x, t)$ thus determined is not smooth but is only continuous with a bounded first derivative.

Of course, we can avoid this difficulty if we first calculate terms in (0.9) and then pass to (0.11). Such a construction, which takes into account the fact that the functions $S_{j}$ and $\varphi_{j}$ can loose smoothness at the points where the Jacobian of projection mapping of the Lagrangian manifold on $R_{x}^{n}$ is zero, was proposed by V. P. Maslov [26] and is ideologically similar to the construction of the Maslov canonical operator (the Fourier integral operators) $[8,10]$. A version of Maslov's construction was proposed in $[28,9]$.

The above procedure is quite well for constructing an asymptotic solution of the Cauchy problem; some estimates of the discrepancy between the asymptotic and exact solutions were also obtained. 
But attempts to apply these constructions to solve the inverse problem meet insurmountable difficulties, and the details of the construction based on the use of integral representations do not play any role here.

The problem consists in information that we need to obtain from the solution at $t=T$ and the use to reconstruct the solution for $0 \leq t<T$.

Indeed, as was shown above, if there are singularities in the projection of the Lagrangian manifold on $R_{x}^{n}$, then the solution can have the form (0.9) at certain points, but as was shown above, some of the terms in this sum are "infinitely" small compared with the other. We can "measure" only expressions of the form (0.11) which do not contain information about the parts of the Lagrangian manifold corresponding to the "infinitely" small terms of the solution. But if we move backwards in time, then these (unknown) parts of the Lagrangian manifold can get into the domains that is uniquely projected on $R_{x}^{n}$, and then they are responsible, in the projection, for the principal part of the solution.

Thus,

(i) If for $t=T$ the function $\phi(x, t)=\lim _{\varepsilon \rightarrow 0}\left(-\varepsilon \ln u_{\varepsilon}\right)$ is smooth, then the principal part of the solution $u_{\varepsilon}$ of the Cauchy problem can be reconstructed for $0 \leq t<T$ in the entire space $R_{x}^{n}$.

(ii) If for $t=T$ the derivatives of the function $\phi(x, t)$ have singularities (discontinuities), then, for $0<t<T$, the principal part of the function $u_{\varepsilon}$ (the solution of the Cauchy problem) cannot be reconstructed in the entire domain, because there are domains where it is impossible to reconstruct the solution.

In our future considerations for non smooth case we will use the relation between transport and continuity equations. This relation between the solutions of the continuity equation and the system consisting of the Hamilton-Jacobi equation plus the transport equation has been well studied before in the case of a smooth action functional.

Let the velocity field $u$ be determined as the family of velocities of points on the projections of the trajectories of the Hamiltonian system corresponding to the Hamilton-Jacobi equation. In this velocity field, as it was mentioned by E. Madelung [13], the squared solution of the transport equation satisfies the continuity equation

$$
\rho_{t}+(\nabla, u \rho)+a \rho=0
$$

with some additional term $a \rho$, which is defined below ( $a$ is equal to 0 if the Hamiltonian is formally self-adjoint). The main obstacle to the extension of this correspondence globally in time is the fact that in 
general the solution of the Hamilton-Jacobi equation are smooth only locally in time. The loss of smoothness is equivalent to the appearance of singularities of the velocity field mentioned above. Till the recent time there was no method for constructing formulas for solutions of continuity equation for a discontinuous velocity field. In Madelung's approach the divergent form of the continuity equation (in difference with transport equation) is very important property allows precisely to introduce a concept of global solution in spite of singularities in the velocity field.

In the present work we generalize Madelung's approach to the case in which the singular support of the velocity field is a stratified manifold transversal to the velocity field trajectories. This holds, for example, in the one-dimensional case under the condition that, for any $t \in[0, T]$, the singular support is a discrete set without limit points.

The class of solutions constructed in this way admits the motion forward and backward in time. Here we discuss only the construction as itself, the invertibility problem we plan to discuss in our next paper.

\section{Generalized solutions of the continuity equation}

Here we follow the approach developed in [1], where the solution is understood in the sense of an integral identity, which, in turn, follows from the fact that relation $(0.12)$ can be understood in the sense of distributional space $\mathcal{D}\left(\mathbb{R}_{x, t}^{n+1}\right)$. The first step in this way has been done in [14], see also [15], [16], where the approach based on smooth approximations of the solutions was used.

We specially note that the integral identities in [1] can be derived without using the construction of nonconservative products $[2,4]$ of the nonsmooth and generalized functions (or measure solutions [5]), and the value of the velocity on the discontinuity lines (surfaces) is not given a priori but is calculated. In the case considered in [1], the integral identities exactly coincide in form with the identities derived using the construction of a nonconservative product (measure solutions) in the situation described at the end of above introduction, which we shall now make more precise.

First, we consider an $n$-1-dimensional surface $\gamma_{t}$ moving in $\mathbb{R}_{x}^{n}$, which is determined by the equation

$$
\gamma_{t}=\{x ; t=\psi(x)\},
$$

where $\psi \in C^{1}\left(\mathbb{R}^{n}\right)$, and $\nabla \psi \neq 0$ in the domain in $\mathbb{R}_{x}^{n}$ where we work. 
This is equivalent to determining a surface by an equation of the form

$$
S(x, t)=0
$$

( $S \in C^{1}$ in both variables, $\left.S(x, t)=0,\left.\nabla_{x, t} S\right|_{S=0} \neq 0\right)$ under the condition that

$$
\frac{\partial S}{\partial t} \neq 0
$$

We remain that the situation with $\frac{\partial S}{\partial t}=0$, can also be covered by making the change of variables $x_{i}^{\prime}=x_{i}-c_{i} t$ with appropriately chosen $c_{i}, i=1 \ldots n$, solving the problem with the moving surface and then returning to the original variables. Possible generalizations are considered later in this section.

Next, we assume that $\zeta$ belongs to $C_{0}^{\infty}\left(\mathbb{R}^{n} \times \mathbb{R}_{+}^{1}\right)$. Then, by definition,

$$
\langle\delta(t-\psi(x)), \zeta(x, t)\rangle=\int_{\mathbb{R}^{n}} \zeta(x, \psi(x)) d x,
$$

where $\delta$ is the Dirac delta function and $\langle$,$\rangle is the distributional pairing$ (with respect to the variable $t \in \mathbb{R}_{+}^{1}$ and $x \in \mathbb{R}^{n}$ ).

Let $\delta(t-\psi(x))$ be applied to the test function $\eta \in C_{0}^{\infty}\left(\mathbb{R}^{n}\right)$, then

$$
\langle\delta(t-\psi(x)), \eta(x)\rangle=\int_{\gamma_{t}} \eta d \omega_{\psi},
$$

where $d \omega$ is the Leray form [6] on the surface $\{t=\psi(x)\}$ such that $-d \psi d \omega_{\psi}=d x_{1} \ldots d x_{n}$.

One can show that (see $[1],[6])$

$$
\langle\delta(t-\psi(x)), \eta(x)\rangle=\int_{\gamma_{t}} \frac{\eta(x)}{|\nabla \psi|} d \sigma .
$$

First, we assume that the solution $\rho$ to Eq. (0.12) has the form

$$
\rho(x, t)=R(x, t)+e(x) \delta(t-\psi(x))
$$

where $R(x, t)$ is a piecewise smooth function with possible discontinuity at $\{t=\psi(x)\}$ :

$$
R=R_{0}(x, t)+H(t-\psi(x)) R_{1}(x, t),
$$

$e \in C\left(\mathbb{R}^{n}\right)$ and has a compact support, $\psi \in C^{2}$ and $\nabla \psi \neq 0$ for $x \in$ $\operatorname{supp} e$, and $H(z)$ is the Heaviside function.

It is clear that the term

$$
e(x) \delta^{\prime}(t-\psi(x))
$$


appears in (0.12) if we differentiate the distribution $\delta(t-\psi(x))$ with respect to $t$. Hence it is necessary to have in $(0.12)$

$$
(\nabla, \rho u)=-e(x) \delta^{\prime}(t-\psi)+\text { smoother summands, }
$$

since $\nabla \delta(t-\psi)=-\nabla \psi \delta^{\prime}(t-\psi)$. Then we must have

$$
\rho u=\frac{e \nabla \psi}{|\nabla \psi|^{2}} \delta(t-\psi)+\text { smoother summands. }
$$

Now we formulate an integral identity, defining a generalized solution to the continuity equation.

We set $\Gamma=\{(x, t) ; t=\psi(x)\}$; this is an $n$-dimensional surface in $\mathbb{R}^{n} \times \mathbb{R}_{+}^{1}$. Let

$$
u(x, t)=u_{0}(x, t)+H(t-\psi) u_{1}(x, t),
$$

where $\psi$ is the same function as before, and $u_{0}, u_{1} \in C\left(\mathbb{R}^{n} \times \mathbb{R}_{+}^{1}\right)$.

Let us consider Eq. (0.12) in the sense of distributions. For all $\zeta(x, t) \in C_{0}^{\infty}\left(\mathbb{R}^{n} \times \mathbb{R}_{+}^{1}\right), \zeta(x, 0)=0$, we have

$$
\left\langle\frac{\partial \rho}{\partial t}+(\nabla, \rho u), \zeta\right\rangle=-\left\langle\rho, \zeta_{t}\right\rangle-\langle\rho u, \nabla \zeta\rangle
$$

Substituting the singular terms for $\rho$ and $\rho u$ calculated above, we come to the following definition.

Definition 1.1 A function $\rho(x, t)$ determined by relation (1.1) is called a generalized $\delta$-shock wave type solution to $(0.12)$ on the surface $\{t=\psi(x)\}$ if the integral identity holds

$$
\begin{gathered}
\int_{0}^{\infty} \int_{\mathbb{R}^{n}}\left(R \zeta_{t}+(u R, \nabla \zeta)+a R \zeta\right) d x d t \\
+\int_{\Gamma} \frac{e}{|\nabla \psi|} \frac{d}{d n_{\perp}} \zeta(x, t) d x=0
\end{gathered}
$$

for all test functions $\zeta(x, t) \in \mathcal{D}\left(\mathbb{R}^{n} \times \mathbb{R}_{+}^{1}\right), \zeta(x, 0)=0, \frac{d}{d n_{\perp}}=\left(\frac{\nabla \psi}{|\nabla \psi|}, \nabla\right)+$ $|\nabla \psi| \frac{\partial}{\partial t}$.

We have also the relation

$$
\int_{\mathbb{R}^{n}} \frac{e}{|\nabla \psi|} \frac{d}{d n} \zeta(x, \psi) d x=\int_{\Gamma} \frac{e}{|\nabla \psi|} \frac{d}{d n_{\perp}} \zeta(x, t) d x .
$$

We note that the vector $n_{\perp}$ is orthogonal to the vector $(\nabla \psi,-1)$, which is the normal to the surface $\Gamma$, i.e., $\frac{d}{d n_{\perp}}$ lies in the plane tangent to $\Gamma$. 
We can give a geometric definition of the field $\frac{d}{d n_{\perp}}$. The trajectories of this vector field are curves lying on the surface $\bar{\Gamma}$, and they are orthogonal to all sections of this surface produced by the planes $t=$ const. Furthermore, it is clear that the expression $\frac{1}{|\nabla \psi|}$ is an absolute value of the normal velocity of a point on $\gamma_{t}$, i.e., on the cross-section of $\Gamma$ by the plane $t=$ const, and the expression $\frac{1}{|\nabla \psi|} \cdot \frac{\nabla \psi}{|\nabla \psi|} \stackrel{\text { def }}{=} \vec{V}_{n}$ is the vector of normal velocity of a point on $\gamma_{t}$. Thus, we have another representation:

$$
\int_{\Gamma} \frac{e}{|\nabla \psi|} \frac{d}{d n_{\perp}} \zeta(x, t) d x=\int_{\Gamma} e\left(\left(\vec{V}_{n}, \nabla\right)+\frac{\partial}{\partial t}\right) \zeta(x, t) d x
$$

where $V_{n}=\pi^{*}\left(v_{n}\right), v_{n}$ is the normal velocity of a point on $\gamma_{t}$, and $\pi^{*}$ is induced by the projection mapping $\pi: \Gamma \rightarrow R_{x}^{n}$.

It follows from the latter definition that the following relations must hold:

$$
\begin{gathered}
R_{t}+(\nabla, R u)+a R \zeta=0, \quad \text { for all points } \quad(x, t) \notin \Gamma, \\
\left([R]-|\nabla \psi|\left[R u_{n}\right]\right)+\left(\frac{d}{d n}\right)^{*} \frac{e}{|\nabla \psi|}=0, \quad \text { for all points } \quad(x, t) \in \Gamma,
\end{gathered}
$$

The last relation can be rewritten in the form

$$
\mathcal{K} E+\frac{d}{d n} E=\left[R u_{n}\right]|\nabla \psi|-[R],
$$

where $E=e /|\nabla \psi|$, the factor $\mathcal{K}=\left(\nabla, \frac{\nabla \psi}{|\nabla \psi|}\right)=\operatorname{div} \nu$ ( $\nu$ is the normal on the surface $\{t=\psi(x)\})$ and, as is known, is the mean curvature of the cross-section of the surface $\Gamma$ by the plane $t=$ const, $\frac{d}{d n}=\left(\frac{\nabla \psi}{\mid \nabla \psi}, \nabla\right)$.

Now we assume that there are two surfaces

$$
\Gamma_{i}=\left\{(x, t) ; t=\psi_{i}(x)\right\}
$$

in $\mathbb{R}^{n} \times \mathbb{R}_{+}^{1}, i=1,2$, whose intersection is a smooth surface

$$
\hat{\gamma}=\left\{(x, t) ;\left(t=\psi_{1}\right) \cap\left(t=\psi_{2}\right)\right\}
$$

belonging to the third surface $\Gamma_{(3)}=\left\{(x, t) ; t=\psi_{3}(x)\right\}$. Further, we assume that the surface $\Gamma_{(3)}$ is a continuation of the surfaces $\Gamma^{(i)}$ in the following sense. We let $n_{\perp}^{(i)}$ denote the curves on the surfaces $\Gamma_{i}$ and we assign that each point $(\hat{x}, \hat{t})$ on the surface $\hat{\gamma}$ is assigned the graph consisting of the trajectories $n_{\perp}^{(1)}$ and $n_{\perp}^{(2)}$ entering $(\hat{x}, \hat{t})$ and the trajectory $n_{\perp}^{(3)}$ leaving this point (i.e., the trajectories $n_{\perp}^{(i)}$ fiber 
the surface $\Gamma^{(i)}$ ). We also assume that the surface (stratified manifold) $\Gamma_{\cup}=\Gamma_{(1)} \cup \Gamma_{(2)} \cup \Gamma_{(3)}$ consists of points belonging to these graphs. Next, we assume that $u(x, t)$ is a piecewise smooth vector field whose trajectories enter $\Gamma_{\cup}$.

Definition 1.2. Let

$$
u(x, t)=u_{0}(x, t)+\sum_{i=1}^{3} H\left(t-\psi_{i}\right) u_{1 i}(x, t),
$$

where $\psi$ is the same function as before, and $u_{0}, u_{1 i} \in C\left(\mathbb{R}^{n} \times \mathbb{R}_{+}^{1}\right)$. The function $\rho(x, t)$ determined by the relation

$$
\rho(x, t)=R(x, t)+\sum_{i=1}^{3} e_{i}(x) \delta\left(t-\psi_{i}(x)\right),
$$

where $R(x, t) \in C^{1}\left(\mathbb{R}^{n} \times \mathbb{R}_{+}^{1}\right) \backslash\left\{\bigcup \Gamma_{t}^{(i)}\right\}$, is called a generalized $\delta$-shock wave type solution to (1.2) corresponding to the stratified manifold $\Gamma_{\cup}$ if the integral identity

$$
\begin{aligned}
\int_{0}^{\infty} & \int_{\mathbb{R}^{n}}\left(R \zeta_{t}+(u R, \nabla \zeta)+a R \zeta\right) d x d t \\
& +\sum_{i=1}^{3} \int_{\Gamma_{(i)}} \frac{e_{i}}{\left|\nabla \psi_{i}\right|} \frac{d}{d n_{\perp}^{(i)}} \zeta(x, t) d x=0
\end{aligned}
$$

holds for all test functions $\zeta(x, t) \in \mathcal{D}\left(\mathbb{R}^{n} \times \mathbb{R}_{+}^{1}\right), \zeta(x, 0)=0, \frac{d}{d n_{\perp}^{(i)}}=$ $\left(\frac{\nabla \psi_{i}}{\left|\nabla \psi_{i}\right|}, \nabla\right)+\left|\nabla \psi_{i}\right| \frac{\partial}{\partial t}$.

As above this relation implies the first equation from (1) outside $\Gamma_{\cup}$, equations of the type of the second equation in (1) on strata $\Gamma_{(i)}$ and the Kirchhoff type relation on $\hat{\gamma}$ :

$$
\left.\left(e_{1}+e_{2}\right)\right|_{\hat{\gamma}}=\left.e_{3}\right|_{\hat{\gamma}} .
$$

Now we consider the case with $\operatorname{codim} \Gamma>1$. First, we note that the second integral in (1.2) can be written as

$$
\int_{\Gamma} \frac{e}{|\nabla \psi|} \frac{d}{d n_{\perp}} \zeta(x, t) d x=\int_{\Gamma} e\left(\left(\frac{\nabla \psi}{|\nabla \psi|^{2}}, \nabla\right)+\frac{\partial}{\partial t}\right) \zeta(x, t) d x .
$$

We note that if the surface $\Gamma$ is determined by the equation $S(x, t)=$ 0 rather than by the simpler equation $\{t=\psi(x)\}$ presented at the beginning of this section, then

$$
\vec{V}_{n}=-\frac{S_{t}}{|\nabla S|} \cdot \frac{\nabla S}{|\nabla S|}=-\frac{S_{t}}{|\nabla S|^{2}} \nabla S
$$


and, of course, the new vector field $\frac{d}{d n_{\perp}}=\left(\vec{V}_{n}, \nabla\right)+\frac{\partial}{\partial t}$ remains tangent to $\Gamma$.

Therefore, in this more general case, using this new vector $\vec{V}_{n}$, we can again rewrite the integral identity from Definition 1.1 as

$$
\int_{0}^{\infty} \int_{\mathbb{R}^{n}}\left(R \zeta_{t}+(u R, \nabla \zeta)+a R \zeta\right) d x d t+\int_{\Gamma} e\left(\left(\vec{V}_{n}, \nabla\right)+\frac{\partial}{\partial t}\right) \zeta(x, t) d x=0 .
$$

This form of integral identity can easily be generalized to the case in which $\Gamma$ is a smooth surface in $\mathbb{R}^{n+1}$ of codimension $>1$.

In this case, instead of $\vec{V}_{n}$, we can use a vector $\vec{v}$ that is transversal to $\Gamma$ and such that the field $(\vec{v}, \nabla)+\frac{\partial}{\partial t}$ is tangent to $\Gamma$. We note that the vector $\vec{v}$ is uniquely determined by this condition, which can be treated as "the calculation of the velocity value on the discontinuity" from the viewpoint of [5] and [7].

Moreover, in this case, the expression for $\rho$ does not contain the Heaviside function, and it is assumed that the trajectories of the field $u$ are smooth, nonsingular outside $\Gamma$, and transversal to $\Gamma$ at each point of $\Gamma$. In this case, the function $\rho$ has the form

$$
\rho(x, t)=R(x, t)+e(x) \delta(\Gamma),
$$

where $R \in C^{1}\left(\mathbb{R}^{n+1} \backslash \Gamma\right), e \in C^{1}(\Gamma)$, and the function $\delta(\Gamma)$ is determined by

$$
\langle\delta(\Gamma), \zeta(x, t)\rangle=\int_{\Gamma} \zeta \omega
$$

where $\omega$ is the Leray form on $\Gamma$. If $\Gamma=\left\{S_{1}(x, t)=0 \cap \cdots \cap S_{k}(x, t)=0\right\}$, $k \in[1, n]$, then $\omega$ is determined by the relation, see [6], p. 274,

$$
d t d x_{1} \cdots d x_{n}=d S_{1} \cdots d S_{k} \omega
$$

In this case, we assume that the functions $S_{k}$ are sufficiently smooth (for example, $C^{2}\left(\mathbb{R}^{n} \times \mathbb{R}_{+}^{1}\right)$ ) and their differentials on $\Gamma$ are linearly independent.

Moreover, we can assume that the inequality

$$
J=\frac{\mathcal{D}\left(S_{1}, \ldots, S_{n}\right)}{\mathcal{D}\left(t, x_{1}, \ldots, x_{n-1}\right)} \neq 0
$$

holds. This inequality is an analog of $S_{t} \neq 0$ at the beginning of this section and allows us to write $\omega$ in the form

$$
\omega=J^{-1} d x_{k} \cdots d x_{n}
$$


The integral identity, an analog of (1.7), has the form

$\int_{0}^{\infty} \int_{\mathbb{R}^{n}}\left(R \zeta_{t}+(u R, \nabla \zeta)+a R \zeta\right) d x d t+\int_{\Gamma} e\left((v, \nabla)+\frac{\partial}{\partial t}\right) \zeta(x, t) \omega=0$.

Integrating the latter relation by parts, we obtain equations for determining the functions $e$ and $R$ similarly to (1.4).

Now we assume that the singular support of the velocity field is the stratified manifold $\bigcup \Gamma_{i}$ with smooth strata $\Gamma_{t}$ of codimensions $n_{i} \geq 1$.

We also assume that the velocity field trajectories are transversal to $\bigcup \Gamma$ and are entering trajectories.

Then the general solution of Eq. (0.12) has the form

$$
\rho(x, t)=R(x, t)+\sum e_{i} \delta\left(\Gamma_{i}\right)
$$

where $R(x, t)$ is a function smooth outside $\bigcup \Gamma_{i}, e_{i}$ are functions defined on the strata $\Gamma_{i}$, and the sum is taken over all strata $\Gamma_{i}$.

The integral identities determining such a generalized solution have the form

$$
\begin{aligned}
\int_{0}^{\infty} & \int_{\mathbb{R}^{n}}\left(R \zeta_{t}+(u R, \nabla \zeta)+a R \zeta\right) d x d t \\
& +\sum_{i} \int_{\Gamma_{i}} e_{i}\left[\left(\left(v_{i}, \nabla\right)+\frac{\partial}{\partial t}\right) \zeta(x, t)\right] \omega_{i}=0 .
\end{aligned}
$$

This implies that, outside $\bigcup \Gamma_{i}$, the function $R$ satisfies the continuity equation

$$
R_{t}+(\nabla, u R)+a R=0
$$

and, on the strata $\Gamma_{j}$ for $n_{j}=1$, equations of the form (1.4) hold, which contain the values of $R$ brought to $\Gamma_{t}$ along the trajectories. For $n_{l}=n-k, k>1$, on the strata $\Gamma_{l}$, we have the equations

$$
\frac{\partial}{\partial t} e_{l} \mu_{l}+\left(\nabla, v_{l} e_{l} \mu_{l}\right)=F_{l} \mu_{l}
$$

where $\mu_{l}$ is the density of the measure $\omega_{l}$ with respect to the measure on $\Gamma_{l}$ which is left-invariant with respect to the field $\frac{\partial}{\partial t}+\left\langle v_{l}, \nabla\right\rangle$, and $F_{l}$ is defined by the following construction. Denote a $\varepsilon$-neighborhood of $\Gamma_{l}$ by $\Gamma_{l}^{\varepsilon}$ and denote its boundary by $\partial \Gamma_{l}^{\varepsilon}$. Let us consider the integral appearing after integration by parts:

$$
\int_{\partial \Gamma_{l}^{\varepsilon}} \zeta \rho u_{n l} \omega_{l}^{\varepsilon}
$$


where $u_{n l}$ is the normal component of velocity $u$ on $\partial \Gamma_{l}^{\varepsilon}, \omega_{l}^{\varepsilon}$ is the Leray measure on $\partial \Gamma_{l}^{\varepsilon}, \zeta$ is a test function. Passing to the limit as $\varepsilon \rightarrow 0$ we obtain

$$
\lim _{\varepsilon \rightarrow 0} \int_{\partial \Gamma_{l}^{\varepsilon}} \zeta \rho u_{n l} \omega_{l}^{\varepsilon}=\int_{\Gamma_{l}} \zeta F_{l} \omega_{l}
$$

It is well known that outside $\bigcup \Gamma_{i}$ the function $R(x, t)$ can be calculated using the famous Cauchy formula

$$
R(x . t)=\rho_{0}(x, t)\left|\frac{D x}{D x_{0}}\right|^{-1} \exp \left(-\int_{0}^{t} a d t^{\prime}\right)
$$

where $\rho_{0}$ ia a constant along the trajectories of the field $\mathrm{u}$ outside $\bigcup \Gamma_{i}$, $\left|\frac{D x}{D x_{0}}\right|$ is the jacobian of the mapping corresponding to the shift along the trajectories of $u$ and the integral under exponent is calculating along the trajectories of the field $u$.

This formula implies that the limit as $\varepsilon \rightarrow 0$ of the above integral exists.

We note that it follows from the above that the function $R$ is determined independently of the values of $v_{i}$ on the strata under the condition that the field trajectories enter $\bigcup \Gamma_{i}$.

In conclusion, we consider the case where the coefficient $a$ has a singular support on $\bigcup \Gamma_{i}$, i.e.,

$$
a=f(u) .
$$

In this case, we set

$$
a \rho=\check{a} \rho+\sum f\left(v_{i}\right) e_{i} \delta\left(\Gamma_{i}\right) .
$$

where $\check{a}=f(u)$ outside $\bigcup \Gamma_{i}$. We note that such a choice of the definition of the term $a \rho$ is not unique in this case. But, first, it is consistent with the common concept of measure solutions (see [3],[5]) and, second, it is of no importance for the construction of the solution outside $U \Gamma_{i}$ for the case in which the trajectories $u$ enter $\bigcup \Gamma_{i}$.

In this case, identity (1.9) takes the form

$$
\begin{aligned}
\int_{0}^{\infty} & \int_{\mathbb{R}^{n}}\left(R \zeta_{t}+(u R, \nabla \zeta)+f(u) R \zeta\right) d x d t \\
& +\sum_{i} \int_{\Gamma_{i}} e_{i}\left[\left(\left(v_{i}, \nabla\right)+\frac{\partial}{\partial t}+f\left(v_{i}\right)\right) \zeta(x, t)\right] \omega_{i}=0
\end{aligned}
$$

and Eq. (1.7) can be rewritten in the form

$$
\frac{\partial}{\partial t}\left(e_{l} \mu_{l}\right)+\left(\nabla, v_{l} e_{l} \mu_{l}\right)+f\left(v_{l}\right)=F_{l} \mu_{l}
$$


All the afore said gives the following statement.

Theorem 1. Let that the following conditions be satisfied for $t \in[0, T]$, $T>0$ :

(1) $\bigcup \Gamma_{i}$ is a stratifies manifold with smooth strata $\Gamma_{i}$;

(2) the trajectories of the field $u$ are smooth outside $\bigcup \Gamma_{i}$, enter $\bigcup \Gamma_{i}$ and do not intersect outside $\bigcup \Gamma_{i}$;

(3) equations (1.13) are solvable on the strata $\Gamma_{i}$;

(4) the Kirchhoff laws are satisfied on the intersections of strata $\Gamma_{i}$.

Then there exist a general solution to the continuity equation (0.1) with $a=f(u)$ in the sense of the integral identity (1.12).

\section{The Maslov tunnel asymptotics}

We recall that the asymptotic solutions of a general Cauchy problem for an equation with pure imaginary characteristics was first constructed by V. P. Maslov [8]. In the present paper, we consider only the following Cauchy problem

$$
-h \frac{\partial u}{\partial t}+P\left(\stackrel{2}{x},-h \frac{1}{\partial x}\right) u=0,\left.\quad u(x, t, h)\right|_{t=0}=e^{-S_{0}(x) / h} \varphi^{0}(x),
$$

where $P(x, \xi)$ is the (smooth) symbol of the Kolmogorov-Feller operator [9], $S_{0} \geq 0$ is a smooth function, $\varphi^{0} \in C_{0}^{\infty}, h \rightarrow+0$ is a small parameter characterizing the frequency and the amplitude of jumps of the Markov stochastic process having having transition probability given by $P(x, \xi)$. To be more precise, we can have in the mind the following form of $P(x, \xi)$ :

$$
P(x, \xi)=(A(x) \xi, \xi)+V(x)+\int_{\mathbb{R}^{n}}\left(e^{i(\xi, \nu)}-1\right) \mu(x, d \nu),
$$

where $A(x)$ is positive definite smooth matrix and $\mu(x, d \nu)$ is a family of bounded measures smooth with respect to $x$. The symbol $P(x, \xi)$ can also depend on $t$, we will be more precise later on.

Locally in $t$, an asymptotic solution of problem (2.1) can be constructed according to the scheme of the WKB method, see [8]: the solution is constructed in the form

$$
u=e^{-S(x, t) / h} \sum_{i=0}^{\infty}\left(\varphi_{i}(x, t) h^{i}\right.
$$


in the sense of asymptotic series. In this case, for the functions $S(x, t)$ and $\varphi_{0}(x, t)$ we obtain the following problems:

$$
\begin{gathered}
\frac{\partial S}{\partial t}+P\left(x, \frac{\partial S}{\partial x}\right)=0,\left.\quad S(x, t)\right|_{t=0}=S_{0}(x), \\
\frac{\partial \varphi_{0}}{\partial t}+\left(\nabla_{\xi} P\left(x, \frac{\partial S}{\partial x}\right), \nabla \varphi_{0}\right)+\frac{1}{2} \sum_{i j} \frac{\partial^{2} P}{\partial \xi_{i} \partial \xi_{j}} \frac{\partial^{2} S}{\partial x_{i} \partial x_{j}} \varphi_{0}=0, \\
\left.\varphi_{0}(x, t)\right|_{t=0}=\varphi^{0}(x),
\end{gathered}
$$

As is known, the solution of problem (2.2) is constructed using the solutions of the Hamiltonian system assumed to exist and to be smooth

$$
\begin{gathered}
\dot{x}=\nabla_{\xi} P(x, p),\left.\quad x\right|_{t=0}=x_{0}, \\
\dot{p}=-\nabla_{x} P(x, p),\left.\quad p\right|_{t=0}=\nabla S_{0}\left(x_{0}\right) .
\end{gathered}
$$

This solution is smooth on the support of $\varphi_{0}(x, t)$ for all $t$ such that the Jacobian $\left|D x / D x_{0}\right| \neq 0$ for $x_{0} \in \operatorname{supp} \varphi^{0}(x)$. We let $g_{H}^{t}$ denote the translation mapping along the trajectories of the Hamiltonian system (2.4).

We recall that the plot

$$
\Lambda_{0}^{n}=\left\{x=x_{0}, p=\nabla S_{0}\left(x_{0}\right)\right\}
$$

is the initial Lagrangian manifold corresponding to Eq. (2.2), and $\Lambda_{t}^{n}=$ $g_{h}^{t} \Lambda_{0}^{n}$ is the Lagrangian manifold corresponding to Eq. (2.2) at time $t$. Let $\pi: \Lambda_{t}^{n} \rightarrow \mathbb{R}_{x}^{n}$ be the projection of $\Lambda_{t}^{n}$ on $\mathbb{R}_{x}^{n}$, which is assumed to be proper. The point $\alpha \in \Lambda_{t}^{n}$ is said to be essential if

$$
\hat{S}(\alpha, t)=\min _{\beta \in \pi^{-1}(\alpha)} \hat{S}(\beta, t)
$$

and nonessential otherwise. Here $\hat{S}$ is the action on $\Lambda_{t}^{n}$ determined by the formula

$$
\hat{S}(\beta, t)=\int_{0}^{t} p d x-H d t,
$$

where the integral is calculated along the trajectories of the system (2.4) the projection of whose origin is $x_{0}=\beta$. As is known

$$
S(x, t)=\hat{S}\left(\pi^{-1} x, t\right)
$$

at regular points where the projection $\pi$ is bijective. 
The global in time asymptotic solution of problem (2.1) is given by the Maslov tunnel canonical operator.

To define this operator, following $[8,10]$ we introduce the set of essential points $\bigcup \gamma_{i t} \subset \Lambda_{t}^{n}$. This set is closed because the projection $\pi$ is proper, i.e. that for all $x$ the set of $p$ such that $(x, p) \in \Lambda_{t}^{n}, \pi(x, p)=x$ is finite.

Suppose that the open domains $U_{j} \subset \Lambda_{t}^{n}$ form a locally finite covering of the set $\bigcup \gamma_{i t}$. If the set $U_{j}$ consists of regular points, then we set

$$
u_{j}=e^{-S_{j}(x, t) / h} \varphi_{0 j}(x, t)
$$

where

$$
\varphi_{0 j}(x, t)=\psi_{0 j}(x, t)\left|\frac{D x_{0}}{D x}\right|^{1 / 2}
$$

where $\psi_{0 j}(x, t)$ being the solution of the equation

$$
\frac{\partial \psi_{0 j}}{\partial t}+\left(P_{\xi}\left(x, \nabla S_{j}\right), \nabla \psi_{0 j}\right)-\frac{1}{2} \operatorname{tr} \frac{\partial^{2} P}{\partial x \partial \xi}\left(x, \nabla S_{j}\right) \psi_{0 j}=0 .
$$

that exists and is smooth whenever $\left|D x / D x_{0}\right| \neq 0$. The solution $u_{j}$ in the domain containing essential (nonregular) points (at which $d \pi$ is degenerate) is given in the following way: the canonical change of variables is performed so that the nonregular points become regular, then we determine a fragment of the solution in new coordinates by formula (2.5) and return to the old variables, applying the "quantum" inverse canonical transformation to the solution obtained in the new coordinates.

The Hamiltonian determining this canonical transformation has the form

$$
H_{\sigma}=\frac{1}{2} \sum_{i=1}^{n} \sigma_{k} p_{k}^{2},
$$

where $\sigma_{1}, \ldots \sigma_{n}=$ const $>0$.

The canonical transformation to the new variables is given by the translation by the time -1 along the trajectories of the Hamiltonian $H_{\sigma}$. One can prove (see [8],[10]) that the family of sets $\sigma$ for which the change of variables takes a regular point into a nonregular is not empty.

Next, the solution near the essential point is determined by the relation

$$
u_{j}=e^{\frac{1}{h} \hat{H}_{\sigma}} \tilde{u}_{j}
$$


where $\tilde{u}_{j}$ is given by formula (2.5) in the new variables and

$$
\hat{H}_{\sigma}=\frac{1}{2} \sum_{k=1}^{n} \sigma_{k}\left(-h \frac{\partial}{\partial x_{k}}\right)^{2} .
$$

On the intersections of singular (containing singular points) and nonsingular charts (without singular points), we must match $S_{j}$ and $\psi_{0 j}$. This can be done by applying the Laplace method to the integral whose kernel is a fundamental solution for the operator $-h \frac{\partial}{\partial t}+\hat{H}_{\sigma}$. This integral appears if we write the right-hand side of (2.7) in detail. In this case, since the solution is real, the Maslov index which is well-known [8] to appear in hyperbolic problems does not appear. The complete representation of the solution of problem (2.1) is obtained by summing functions of the type (2.5) and (2.7) over all the domains $U_{j}$, for more detail, see [8], [10].

The asymptotics thus constructed is justified, i.e., the proximity between the exact and asymptotic solutions of the Cauchy problem (2.1) is proved $[8,9]$. More precisely it is proved that at the points of the set $\pi\left(\bigcup \gamma_{i t}\right.$ where the projection $\pi$ is bijective the following estimate holds:

$$
u(x, t, h)-u_{j}=O(h)
$$

In the preceding case we noted that values of the solution of the continuity equation at nonregular points are independent of the values of the solution on the singularity support (of course, the inverse influence takes place) by the condition that the velocity field trajectories enters the singular support.

In the case of the canonical operator construction briefly described above, the relation between the solutions at essential and nonessential point is also unilateral, namely, the essential points are "bypassed" using (2.7), but the values of the functions $\tilde{\psi}_{o j}$ contained in $\tilde{u}_{j}$ on the singularity support do not determine the values at the regular points (but the converse is not true).

Now we note that the function $S(x, t)$ such that

$$
\left.S(x, t)\right|_{U_{j}}=S_{j}\left(\pi^{-1}(\alpha), t\right)
$$

is globally determined and continuous at points of the domain $\pi\left(\bigcup \gamma_{i t}\right) \subset$ $\mathbb{R}_{x}^{n}$. We denote this set by $\bigcup \Gamma_{i}$ and assume that this is a stratified manifold with smooth strata $\Gamma_{i t}$ of different codimensions. We note that, for example, if the inequality $\nabla\left(S_{i}(x, t)-S_{j}(x, t)\right) \neq 0$ holds while we pass from one branch $\Lambda_{t}^{n} \cap \bigcup \gamma_{i t}$ to another, then the set $\pi\left\{\left(\tilde{S}_{i}-\tilde{S}_{j}\right)=0\right\}$ generates a smooth stratum of codimension 1 . In the one-dimensional 
case, all strata are points or curves on the $(x, t)$-plane (under the above assumptions about the singularities being discrete).

Now we consider the equation for $\psi_{0 j}^{2}$. We denote this function by $\rho$ and then obtain

$$
\frac{\partial \rho}{\partial t}+(\nabla, u \rho)+a \rho=0
$$

where $u(x, t)=\nabla_{\xi} P(x, \nabla S)$ and $a=-\operatorname{tr} \frac{\partial^{2} P}{\partial x \partial \xi}(x, \nabla S)$.

If the condition

$$
\operatorname{Hess}_{\xi} P(x, \xi)>0
$$

is satisfied, then it follows from the implicit function theorem that $\nabla S(x, t)=F(x, u(x, t))$, where $F(x, u)$ is a smooth function and

$$
a=f(x, u),
$$

where $f(x, z)$ is again a smooth function.

Let us return to the formula (1.8) and denote the regular (in the sense of distributions) part of $\rho$ by $\rho_{\text {reg }}$.

Thus, we can formulate the following theorem.

Theorem 2. Suppose that the following conditions are satisfied for $t \in$ $[0, T], T>0$ :

(1) There exists a smooth solution of the Hamiltonian system (2.4).

(2) The singularities of the velocity field $u=\nabla_{\xi} P(x, \nabla S)$ form a stratified manifold with smooth strata and $\operatorname{Hess}_{\xi} P(x, \xi)>0$.

(3) There exists a generalized solution $\rho$ of the Cauchy problem for Eq. (2.8) in the sense of the integral identity (1.10).

Then at the points of $\pi\left(\bigcup \gamma_{i t}\right)$ where the projection $\pi$ is bijective, the asymptotic solution of the Cauchy problem (2.1) has the form

$$
u=\exp (-S(x, t) / h)\left(\sqrt{\rho_{\text {reg }}}+O(h)\right) .
$$

This theorem is a global in time analog of the corresponding Madelung observation about local solutions of Schroedinger type equations.

Now we demonstrate the connection between solutions of continuity and transport equations. It is easy to see that by construction a transport equation solution is equal to $\rho_{\text {reg }}$ out of the points of singular support. More that one can define $\sqrt{\rho}$ globally in distributional sense and thus defined square root is equal to $\rho_{\text {reg. }}$. To prove this statement it is sufficient to note that $\delta$-shock type solution to continuity equation in the form $(1.8)$ can be obtained $[14,15])$ as a weak limit of a weak asymptotic solution of the form

$$
\rho(x, t)=\rho_{\mathrm{reg}}+\sum e_{i} \delta_{\varepsilon}\left(\Gamma_{i}\right),
$$


where $x \in R^{n}, \varepsilon$ is an axillary small parameter and $\delta_{\varepsilon}$ is a regularization of $\delta$-function of the form

$$
\delta_{\varepsilon}=\varepsilon^{-\left(n-n_{i}\right)} \omega\left(S_{i} / \varepsilon\right)
$$

Here $S_{i}$ are smooth functions such that $\left.\nabla S_{i}\right|_{S_{i}=0} \neq 0$ and the equation $S_{i}=0$ defines the strata $\Gamma_{i}, n_{i}=\operatorname{dim} \Gamma_{i}$ and $\omega=\omega(\eta)$ belongs to the Schwartz space of test functions. Without loss of generality one can consider the case $n_{i}=0$ in $(x, t)$ half-plain for each fixed $t$. In this case the function $S_{i}$ can be chosen in the form $S=x-\phi(t)$ and our statement becomes

$$
\lim _{\varepsilon \rightarrow 0} \int_{R^{1}}\left(\sqrt{\left(\rho_{\mathrm{reg}}+e_{i} \varepsilon^{-1} \omega((x-\phi) / \varepsilon)\right)}-\sqrt{\rho_{\mathrm{reg}}}\right) \varphi(x) d x=0,
$$

for arbitrary test function $\varphi(x)$ from $C_{0}^{\infty}$.

After the change of variables $\eta=(x-\phi) / \varepsilon$ the $(2.10)$ gets the equivalent form

$$
\lim _{\varepsilon \rightarrow 0} \sqrt{\varepsilon} \int_{R^{1}}\left(\sqrt{\varepsilon \rho_{\mathrm{reg}}+e_{i} \omega(\eta)}-\sqrt{\varepsilon \rho_{\mathrm{reg}}}\right) d \eta=0,
$$

which is obviously true under the trivial inequality $\sqrt{1+z} \leq 1+z / 2$. Indeed consider the domain in $R_{\eta}^{1}$ where $\omega^{\alpha}<\rho \varepsilon$ for some $\alpha, 0<\alpha<1$. Then the mentioned inequality gives the estimate $O\left(\varepsilon^{1 / 2}\right)$ for the integral under limit in (2.11). In the case $\omega^{\alpha} \geq \rho \varepsilon$, because the function $\omega$ is decreasing (belongs to the Schwartz space), the measure of corresponding domain can be estimated as $(\rho \varepsilon)^{-1 / N}$ where $N$ is arbitrary positive number. This completes the proof.

\section{Particular cases}

The theorem stated in the previous section requires that some assumptions are satisfied. The most restrictive is the item 3 in the theorem above-that is the existence of the global generalized solution to continuity equation. Under the above made assumptions it is possible to construct this solution using characteristics, but only in the case where the structure of singular support of $u$ is not changing in time-all sections of the stratified manifold introduced above by planes $t=$ const are diffeomorphic. A more complicate situation arises, when the singularities of the velocity field change their structure. In this case the problem of the construction of a global in time generalized solution to the continuity equation has not been solved yet. The obstacle is that in this 
case usually one has no global in time expression for the velocity field $u$. In turn this does not allow to apply formula (1.11) to construct global solution to the continuity equation. In multy-dimensional case as far as we know there is only one result concerning to shock wave generation [14] which allows to construct global in time approximation of the shock wave formation process. But this is slight different from the construction that we needs here. In the one dimensional case the situation is better and we have all needed formulas.

We begin with the spatially homogeneous case. Here the problem is equivalent to the one of constructing a formula for a global solution to conservation law equation

$$
\frac{\partial v}{\partial t}+\frac{\partial P(v, t)}{\partial x}=0
$$

Here $P\left(-h \frac{\partial}{\partial x}, t\right)$ is the same operator as in (2.1) but assumed to be independent of $\mathrm{x}$ with the symbol $P(\xi, t)$ and $v=\partial S / \partial x$. The velocity field $u$ in this case is $P_{\xi}(v, t)$. In [11] a construction of the global solution to the continuity equation where the velocity field is given by the solution of the equation (3.1) was given. Because the set of singular points is discrete by our assumptions, without loss of generality one can consider the case were only one point of singularity appears. Denote the corresponding (smooth) initial condition by $u_{0}$, the instant where the singularity appears by $t^{*}$ and the point of singularity by $x^{*}$.

The first step of construction suggested in [11], [12] (see also [18]) is that we change $u_{0}$ in a small neighborhood of of origin $x_{0}^{*}$ of the trajectory coming to $x^{*}$ when $t=t^{*}$. We denote this new part of initial data as $u_{1}\left(x_{0}\right)$ for $x_{0} \in\left(x_{0}^{*}-\beta, x_{0}^{*}+\beta\right), \beta \rightarrow 0$ and assume

$$
\varepsilon \beta^{-1} \rightarrow 0, \quad \varepsilon \rightarrow 0
$$

We define the function $u_{1}=u_{1}\left(x_{0}, t\right)$ as a solution of implicit equation

$$
P_{\xi}^{\prime}\left(u_{1}, t\right)=-K(t) x_{0}+b(t)
$$

The latter equation is solvable under the condition $\operatorname{Hess}_{\xi} P(x, \xi)>0$, formulated above.

The functions $K(t)$ and $b(t)$ are defined from the condition of continuity of the characteristics flow, i.e

$$
u_{1}\left(x_{0}^{*}-\beta, t\right)=u_{0}\left(x_{0}^{*}-\beta, t\right), \quad u_{1}\left(x_{0}^{*}+\beta, t\right)=u_{0}\left(x_{0}^{*}+\beta, t\right)
$$

It is easy to check that this choice of $u_{1}$ provides that the Jacobian $\left|D x / D x_{0}\right|$ is identically equal to 0 for $t=t^{*}$ and $x_{0} \in\left(x_{0}^{*}-\beta, x_{0}^{*}+\right.$ 
$\beta$ ). Here we remove from usual topological concept of general position considering the situation of identical equality that can be destroyed by small perturbation. But this construction follows from the algebraic concept and allows to present the solution of (3.1) in the form of linear combination of Heaviside functions (see [11]).

The second step of our construction of an approximation is a modification of the definition of characteristics. We set

$$
\dot{x}=(1-B) P_{\xi}^{\prime}\left(u_{1}\left(x_{0}, t\right), t\right)+B c, \quad x_{0} \in\left(x_{0}^{*}-\beta, x_{0}^{*}+\beta\right)
$$

and

$$
\dot{x}=P_{\xi}^{\prime}\left(u_{0}, t\right),
$$

where $x_{0}$ does not belong to $\left(x_{0}^{*}-\beta, x_{0}^{*}+\beta\right)$,

$$
c=\frac{P\left(v\left(x\left(x_{0}^{*}+\beta, t\right), t\right)\right)-P\left(v\left(x\left(x_{0}^{*}-\beta, t\right), t\right)\right)}{v\left(x\left(x_{0}^{*}+\beta, t\right), t\right)-v\left(x\left(x_{0}^{*}-\beta, t\right), t\right)}
$$

Initial data for (3.4) are the following:

$$
\left.x\right|_{t=0}=x_{0}+A \varepsilon, \quad \varepsilon>0 .
$$

The function $\mathrm{B}$ in (3.4) has the form $B=B\left(\left(t-t^{*}\right) / \varepsilon\right)$ and $\mathrm{B}(\mathrm{z})$ is smooth, monotone and increasing from 0 to 1 for $z \in(-\infty, \infty)$. Similarly to [11], [12] one can prove that there exist an $A=$ const such that the Jacobian $\left|D x / D x_{0}\right|$ calculated using the above introduced characteristics is not equal to zero, but it is of order $O(\varepsilon)$ when $t \geq t^{*}+O(\beta)$ when $x_{0} \in\left(x_{0}^{*}-\beta, x_{0}^{*}+\beta\right)$. Using the velocity field generated by $\dot{x}$ we can construct global in time (smooth) solution of the continuity equation in the form (1.11). After that, passing to the limit as $\varepsilon \rightarrow 0$ we will obtain the generalized solution of the continuity equation in the sense of definition from Sec.1 just like it was done in [12].

Spatially inhomogeneous one dimensional case.

We will follow the scheme introduced above. The case under consideration can be treated in the same way as the previous one with modifications. Firstly, we will assume that the symbol $P=P(x, \xi)$ does not depend on $t$. In this case this assumption (which means that the mapping $g_{P}^{t}$ is invertible)will be used to construct the insertion to initial data. In the previous case we did it using the implicit function theorem, see (3.3).

Let $\Lambda_{0}^{1}$ be a smooth nonsingular (w.r.t the projection $\pi$ ) curve in the $(x, p)$ space, which is a Lagrangian manifold corresponding to initial 
data for our problem. We consider Lagrangian manifold $\Lambda_{t^{*}}^{1}=g_{P}^{t^{*}} \Lambda_{0}^{1}$ and assume that there is only one point singular with respect to projection on $x$-axis and its projection is $x^{*}$. Let $\beta$ be the same as above. Let us set $t_{1}^{*}=t^{*}+\beta$. Because of the assumption that $P_{\xi \xi}^{\prime \prime}$ is positive, we have that for $t=t_{1}^{*}$ the Lagrangian manifold $\Lambda_{t_{1}^{*}}^{1}$ has two parts which contain essential points and these parts form a shock wave type curve with the jump at the point $x_{1}^{*}$ where $S_{\text {left }}\left(x_{1}^{*}, t_{1}^{*}\right)=S_{\text {right }}\left(x_{1}^{*}, t_{1}^{*}\right)$. We connect these parts by a vertical line and thus obtain a new Lagrangian manifold, which is piecewise smooth continuous curve with two angle points (ends of the vertical part, the distance between them of order $\beta$ ). We denote this manifold by $\hat{\Lambda}_{t_{1}^{*}}^{1}$ and apply the mapping $g_{P}^{-t_{1}}$ for sufficiently small $t_{1}$ to this manifold. This mapping obviously exists and is a diffeomorpfism because our Hamiltonian $P$ does not depend on $t$. We consider the obtained manifold $g_{P}^{-t_{1}} \hat{\Lambda}_{t_{1}^{*}}^{1}$ as the new Lagrangian manifold corresponding to our problem for $t=t_{1}^{*}-t_{1}$ changing the manifold $\Lambda_{t_{1}^{*}-t_{1}}^{1}$ by $g_{P}^{-t_{1}} \hat{\Lambda}_{t_{1}^{*}}^{1}$. As it was said above the latter manifold is piecewise smooth curve with two angle points and all points of the curve outside of the part between these angle points are regular. Moreover there exist a sufficiently small $t_{1}$ such that the part of the curve between these angle points contains only regular points-these statements are the consequence of the positivity of $P^{\prime \prime}$, its stationarity and the possibility to choose $t_{1}$ small enough (and independent on $\varepsilon$ ).

Denote the projections of the mentioned above angle points on the manifold $g_{P}^{-t_{1}} \hat{\Lambda}_{t_{1}^{*}}^{1}$ to the $x$-axis by $a_{1}<a_{2}$ and note that $\left|a_{1}-a_{2}\right|$ is of order $\beta$.

Like in the previous example we introduce the new characteristics system

$$
\begin{gathered}
\dot{x}=(1-B) P_{\xi}^{\prime}\left(x\left(x_{0}, t\right), p\left(x_{0}, t\right)\right)+B c, \\
\dot{p}=-(1-B) P_{x}^{\prime}\left(x\left(x_{0}, t\right), p\left(x_{0}, t\right), \quad x_{0} \in\left(a_{1}, a_{2}\right),\right.
\end{gathered}
$$

and

$$
\begin{gathered}
\dot{x}=P_{\xi}^{\prime}(x, p), \\
\dot{p}=-P_{x}^{\prime}(x, p)
\end{gathered}
$$

when $x_{0}$ does not belong to $\left(a_{1}, a_{2}\right)$. We have set

$$
c=\frac{P\left(v\left(x\left(a_{2}, t\right), p\left(a_{2}, t\right)\right)\right)-P\left(x\left(a_{1}, t\right), p\left(a_{1}, t\right)\right)}{p\left(x\left(a_{2}, t\right), t\right)-p\left(x\left(a_{1}, t\right), t\right)}
$$

Initial data for (3.5), (3.6) are the following: 


$$
\begin{gathered}
\left.x\right|_{t=0}=x_{0}+A \varepsilon, \\
\left.p\right|_{t=0}=p_{0}\left(x_{0}\right),
\end{gathered}
$$

where $\left(x_{0}, p_{0}\left(x_{0}\right)\right)=g_{P}^{-t_{1}} \hat{\Lambda}_{t_{1}^{*}}^{1}$. The expression in the right hand side of (3.7) is the direct analog of the well known Rankine-Hugoniot expression for the velocity of the shock propagation. In the case under consideration it is the velocity of the point $\check{x}$ on $x$-axis, where $S_{\text {left }}(\check{x}, t)=S_{\text {right }}(\check{x}, t)$.

By the assumption we have only one singular point if we are considering the family of manifolds $\Lambda_{t}^{1}, t \in\left[0, t^{*}\right]$. We also have by construction that the jacobian $J=D x / D x_{0}$ calculated using the solutions of the system (3.5) is not equal to zero. More precisely we have

$$
\lim _{\varepsilon \rightarrow 0} J=H\left(t^{*}-t\right) J_{0},
$$

where $J_{0}$ is the Jacobian calculated using the solutions of (3.5) for $B=0$ $\left(J_{0}=0\right.$ when $t=t^{*}$ by construction) and

$$
J \geq H\left(t^{*}-t\right) J_{0}+C \varepsilon,
$$

where $C=$ const $>0$. This statement directly follows from (3.5) if we take the properties of the function $B$ into account. It means that the velocity field, generated by projections of the solution of the system (3.5), (3.6) on the $x$-axis has nonintersecting trajectories for $\varepsilon>0$. Thus we can use it to construct solutions of the continuity equation. It remains to note that just like in [12] it is easy co check the the limits of these solutions will satisfy to the integral identities introduced in Sec. 1 as the definition of generalized solutions to continuity equation.

\section{References}

[1] V. G. Danilov, "On singularities of continuity equations," Nonlinear Analysis; Theory, Methods and Applications, 68, 6, 1640-1651, (2008), Preprint 2006-41, http://www.math.ntnu.no/coservation/2006

[2] P. G. Le Floch, "An existence and uniqueness result for two nonstrictly hyperbolic systems," in Nonlinear Evolution Equations that Change Type (Springer, Berlin, 1990), pp. 126-138.

[3] G. Dal Maso, P. G. Le Floch, and F. Murat, "Definition and weak stability of nonconservative products," J. Math. Pure Appl. 74, 483-548 (1995). 
[4] A. I. Volpert, "The space BV and quasilinear equations," Math. USSR Sb. 2, 225-267 (1967).

[5] Hanchun Yang, "Riemann problem for a class of coupled hyperbolic systems of conservation laws," J. Diff. Equations 159, 447-484 (1999).

[6] I. M. Gelfand and G. E. Shilov, Generalized Functions (Academic Press, New York, 1964), Vol. 1, (translated from the Russian).

[7] Wanchung Sheng and Tong Zhang, "The Riemann problem for the transportation equation in gas dynamics," Memories of AMS 137 (64), 1-77 (1999).

[8] V. P. Maslov, Asymptotic Methods and Perturbation Theory (Nauka, Moscow, 1988).

[9] V. G. Danilov and S. M. Frolovitchev, "Exact asymptotics of the density of the transition probability for discontinuous Markov processes," Math. Nachrichten 215 (1) 55-90 (2000).

[10] V. P. Maslov and V. E. Nazaikinskii, "Tunnel canonical operator in thermodynamics," Funktsional. Anal. i Prilozhen. 40 (3), 12-29 (2006).

[11] E . Madelung, "Quantentheorie in hydrodynamischer form," Z. Phys. 40, 322 (1926).

[12] V. G. Danilov, "Remarks on the formation and decay of multidimensional shock waves," Preprint 2004-32, http://www.math.ntnu.no/coservation/2004

[13] S. Albeverio, V. M. Shelkovich, "On delta shock front problem," in book: Analytical Approach to Multibalance Laws, Ch. 2 (Ed.O.Rosanova), Nova Science Publishers Inc., 2005, pp.45-88

[14] V. G. Danilov and V. M. Shelkovich, "Delta- shock waves type solutions of hyperbolic systems of conservation law," Quarterly of Appl. Math., 63 (3) 401-427 (2000).

[15] V. G. Danilov, V. M. Shelkovich, "Dynamics of propagation and interaction of delta-shock waves in conservation law systems", Journal of Differential Equations, 211, no. 2, (2005), 333-381. 
[16] S. Albeverio and V. Danilov, "Global in time asymptotics to Kolmogorov-Feller equations," Proc. Conf. Asymptotic Methods and Mathematical Physics, p. 9, Moscow, 05.2010, www.ammp2010.ru

[17] S. Albeverio and V. Danilov, "Global in Time Madelung Transformation for Kolmogorov-Feller Pseudodifferential Equations," arXiv:0904.1945[math-ph]

[18] V. Danilov, "Generalized solutions describing singularity interaction," Int. J. Math. Math. Sci. 29, No. 8, 481-494 (2002).

[19] V. Danilov, "On singularities of continuity equation solutions," Nonlinear Anal. 68, No. 6, 1640-1651 (2008).

[20] V. Danilov and D. Mitrovic, "Weak asymptotics of shock wave formation process," Nonlinear Anal. 61, No. 4, 613-635 (2005).

[21] V. G. Danilov and D. Mitrovic, "Delta shock wave formation in the case of triangular hyperbolic system of conservation laws," J. Differential Equations 245, No. 12, 3704-3734 (2008)

[22] V. Danilov and S. Frolovitchev, "Exact asymptotics of the density of the transition probability for discontinuous Markov processes," Mathematische Nachrichten 215, No. 1, pp. 55-90.

[23] Yu. Kifer, "On the asymptotics of transition density of processes with small diffusion," Theory Probab. Appl. 21, No. 1, 513-522 (1976).

[24] M. Freidlin and A. Wentzell, Random Perturbations of Dynamical Systems, Springer Verlag, New York, 1984; 1998.

[25] V. N. Kolokoltsov and V. P. Maslov, Idempotent analysis and its applications. Translation of Idempotent analysis and its application in optimal control (Russian), "Nauka" Moscow, 1994. Translated by V. E. Nazaikinskii. With an appendix by Pierre Del Moral. Mathematics and its Applications, 401. Kluwer Academic Publishers Group, Dordrecht, 1997.

[26] V. Maslov, "Global exponential asymptotic behavior of solutions of the tunnel equations and the problem of large deviations," (Russian) International conference on analytical methods in number theory and analysis (Moscow, 1981). Trudy Mat. Inst. Steklov. 163 (1984), pp. 150-180. 
[27] V. Maslov and M. Fedoryuk, Semiclassical approximation in quantum mechanics, Reidel, Dordrecht, 1981 (Transl. from the Russian).

[28] V. G. Danilov, "A Representation of the Delta Function via Creation Operators and Gaussian Exponentials and Multiplicative Fundamental Solution Asymptotics for Some Parabolic Pseudodifferential Equations," Russian J. Math. Phys. 3, No. 1 p. 25 (March 1995). 